CDD: 149.946

\title{
DA COSTA ON ONTOLOGY: A NATURALISTIC INTERPRETATION
}

\author{
ANTONIO MARIANO NOGUEIRA COELHO \\ Departamento de Filosofia \\ Universidade Federal de Santa Catarina \\ Campus Trindade, 88010-970 \\ Florianópolis, $S C$ \\ BRASIL \\ acoelho@cfh.ufsc.br
}

\begin{abstract}
Costa's conception of being modifies that of Quine to incorporate relativization to non-classical logics. A naturalistic view of this conception is discussed. This view tries to extend to logic some ideas of Maddy's naturalism concerning mathematics.
\end{abstract}

Keywords: da Costa. Ontology. Maddy's naturalism.

Da Costa's conception of being, in his own words "may be summarized as follows: To be is to be the value of a variable in a specific language with a given underlying logic" (da Costa (2002, p. 279)). This stand, of course, is a variant of that of Quine, to be is to be the value of a variable, incorporating the possibility of relativization to some non-classical logic, that is, relativization to some logic other than the classical first order predicate calculus. For da Costa (2002) there are two kinds of relevance a subject can have to philosophy. These are: direct relevance and indirect relevance. According to him, logic does not have any direct relevance to philosophy; in particular, logic does not have any direct relevance to ontology. By this he means that logic is, in a certain sense, neutral. The logician's research activities are independent of any philosophical doctrine, and in particular of any 
ontological commitment. If pressed by the assertion

that a given development of logic implies (or depends on) some philosophical hypothesis, one can always answer that the development in question constitutes simply a façon de parler, that it does not in fact get involved with any philosophical thesis at all, but that it only feigns to this end. For example, when our attention is called to the Platonist bias of extant set theory, the most obvious reply is that we are working as if Platonism were true, feigning to give countenance to Platonism, but that really we stay above such criticisms. (da Costa (2002, p. 281))

Quite different is the situation of the indirect relevance of logic to philosophy, understood as the interplay between logic and philosophy when logic is supplemented by philosophical principles; this supplementation meaning the consideration of logic from some philosophical point of view. This kind of relevance manifests itself in a multitude of philosophical, in particular ontological, problems, concerning, for instance, the interpretation of Gödel's incompleteness theorems, of LöwenheimSkolem theorems, and, of course, of non-classical logics. Although, as I said, da Costa denies the direct import of logic to philosophy, he sustains that logic "has really an important indirect relevance to philosophy, and especially to ontology" (da Costa (2002, p. 280)). Da Costa understands that those who deny even the indirect relevance of logic to ontology are mainly motivated by a belief in the certainty and a priori character of logic. Being certain and independent of observations, logic would not be about the world. Logic would be anontological, a term employed by Berry in his paper "Logic with Platonism" (1975), meaning that logic has no relation at all with ontology.

For da Costa the certainty of logic is not complete and logic, also, is not entirely a priori. That certainty is not complete, or, at least, that 
we are not certain that certainty is complete is a lesson the paradoxes, da Costa says, and we can add the difficulties related, for instance, to the axiomatization of set theory, a kind of grand logic, gave to us. One of the motivations of Zermelo to axiomatize set theory was to cope with the objections to his proof of the well-ordering theorem, more specifically to the use he made of the axiom of choice in this proof. Zermelo was very successful in axiomatizing set theory and in defending the axiom of choice, but, arguably, success is one thing and certainty is another. Concerning the a priori character, if we, like da Costa, interpret logic as "the most general part of science" (da Costa (2002, p. 283)), then logic is not entirely a priori, or, again, we cannot be sure that logic is entirely a priori. It is, perhaps, submitted to pragmatic constraints originating in the development of the natural sciences. This naturalistic position can affect in different ways the view of the role of logic in ontology. Putting away the supposed certainty and $a$ priori character of logic, da Costa embraces the indirect relevance of logic to ontology and defends his already presented conception of being as relative to an underlying logic, by considering paraconsistent logic and "an inconsistent but apparently non-trivial set theory $Z F^{*}$, which reflects Cantor's Absolute better than any of the extant set theories" (da Costa (2002, p. 287)). By a theory of Cantor's Absolute, da Costa understands "a set theory capturing most of the properties of sets, as they appear in our intuitive and naive handling of them" (da Costa (2002, p. 284)). I will consider another way of defending da Costa's conception of being. A way that is also naturalistic in a certain sense. A sense, I believe, that reflects the naturalism of Penelope Maddy regarding mathematics. To understand this let's go back to Quine. He once wrote that 
Pure mathematics, in my view, is firmly imbedded as an integral part of our system of the world. Thus my view of pure mathematics is oriented strictly to application in empirical science. Parsons has remarked, against this attitude, that pure mathematics extravagantly exceeds the needs of application. It does indeed, but I see these excesses as a simplistic matter of rounding out. We have a modest example of the process already in the irrational numbers: no measurement could be too accurate to be accommodated by a[n] [ir]rational number, but we admit the extras to simplify our computations and generalizations. Higher set theory is more of the same. I recognize indenumerable infinites only because they are forced on me by the simplest known systematizations of more welcome matters. Magnitudes in excess of such demands, e.g., $\beth_{\omega}$ or inaccessible numbers, I look upon only as mathematical recreation and without ontological rights. Sets that are compatible with ' $V=L$ ' in the sense of Gödel's monograph afford a convenient cut-off. (Quine (1986, p. 400)).

Let's remember some points. $V$ is the class of all sets. It splits into the von Neumann hierarchy of sets $V_{\alpha}$ indexed by the ordinal numbers. $V_{0}$ is the empty set; $V_{\alpha+1}$ is the power set of $V_{\alpha}$, and if $\lambda$ is a limit ordinal, then $V_{\lambda}$ is the union of the $V_{\beta}$, where $\beta$ is a member of $\lambda$. $L$ is the constructible universe. It also splits into a hierarchy, now of sets $L_{\alpha}$, indexed by the ordinal numbers. The difference between the von Neumann hierarchy and the constructible hierarchy is that $L_{\alpha+1}$ is the set of subsets of $L_{\alpha}$ first order definable over $L_{\alpha}$ using parameters from $L_{\alpha}$. The constructible universe is an inner model of set theory. " $V=L "$ is the so called axiom of constructibility. It says that every set is constructible. This axiom was introduced by Gödel to prove the consistency of the generalized continuum hypothesis with the axioms 
of set theory. If we add the axiom of constructibility to $Z F$, we obtain a powerful theory that settles most of the questions left open in $Z F C$. The generalized continuum hypothesis and, hence, the axiom of choice are theorems of this theory. The beth-numbers are specified in the following way: $\beth_{0}=\aleph_{0}, \beth_{\alpha+1}$ is the cardinal of the power set of $\beth_{\alpha}$; and if $\lambda$ is a limit ordinal, then $\beth_{\lambda}$ is the supremum of the $\beth_{\alpha}$, where $\alpha$ is a member of $\lambda$. The generalized continuum hypothesis is equivalent to say that for every ordinal $\alpha$ we have $\beth_{\alpha}=\aleph_{\alpha}$. The definition of the beth-numbers makes sense in $Z F C$, that is, we can prove in $Z F C$ that each beth-number exists.

In above quotation, Quine accepts, at least as a matter of convenience, the incorporation of the axiom of constructibility to $Z F$. At the same time he denies ontological rights to $\beth_{\omega}$, whose existence can be proved in the weaker theory $Z F C$. This position is derived from the combination of the indispensability argument for mathematical realism, $\beth_{\omega}$ is not used in applied mathematics, so it has no ontological rights, with the pursuit of ontological economy. The strangeness of this state of affairs was noted, for instance, by Penelope Maddy in her book Naturalism in Mathematics (1997, pp. 106-107). She strongly opposes to the ontological subordination of mathematics, set theory in particular, to natural sciences as Quine defends. Maddy emphasizes that this subordination is in conflict with the practice of mathematics- almost every set theorist rejects the axiom of constructibility, that is, rejects the adoption of $Z F+V=L$ as the standard set theory (of course every set theorist accepts the axiom of constructibility as an instrument to prove consistency results). According to Maddy's mathematical naturalism "mathematics is not answerable to any extra-mathematical tribunal and not in need of any justification beyond proof and the axiomatic method" (Maddy (1997, p. 184)). She understands proof and axiomatic method "as short-hand for the actual methods of mathematics" (Maddy (1997, p. 184)). 
In this spirit I will sketch an intra-mathematical defense of da Costa's logical relativity of being by considering the role non-classical logics play in forcing constructions of models of set theory. The method of inner models that Gödel used when he conceived the constructible universe to prove the consistency of the continuum hypothesis can not be successful to prove the consistency of the negation of the continuum hypothesis. Putting aside some technical points, we can roughly describe the situation as follows: if we could construct, in set theory, a (class) model of set theory in which the negation of the continuum hypothesis were true, then the minimal character of the constructible universe would assure us that this model includes the constructible universe. But the negation of the continuum hypothesis holds in the model and the continuum hypothesis holds in the constructible universe. The proofs of these results being done in set theory. So we could prove in set theory that the model in question is not the constructible universe. This amounts to a proof, in set theory, of the negation of the axiom of constructibility, but this is impossible because this axiom is consistent with set theory (see, for instance, Smullyan and Fitting (1996, p. 189)). To overcome this problem, the method of forcing, invented (or discovered) by Paul Cohen, tells us, among other things, how to generalize the notion of model of set theory (see Jech (1997, p. 137) and Kunen (1980, p. 234)). This generalization meaning the adoption of the semantics of some non-classical logic and the construction of a corresponding nonclassical inner model where the negation of the continuum hypothesis holds, This done, the unprovability of the continuum hypothesis in set theory equipped with classical logic is granted by an appropriate translation from classical logic to the non-classical logic whose semantics was employed (see Smullyan and Fitting (1996, pp. 189-190)). The method of forcing also allows us to obtain classical models, but even in this case some non-classical logic is important to control the properties of the classical model (see, again, Smullyan and Fitting (1996), p. 190). 
We started with Quine's position, to be is to be the value of a variable. If we consider the ontology of formal theories, like $Z F C$, the values of the variables are in the domains of the models of these theories. If to deal with a highly relevant problem of the mathematical practice, the continuum problem, we must, for mathematical reasons, use some non-classical logic and this non-classical logic works extending the domains of the models of the theory related to the problem, then it seems that in fact to be is to be the value of a variable with respect to an underlying logic, as da Costa says. Of course, the method of forcing can be viewed only syntactically, but if we restrict ourselves to the realm of syntax, then the problem is not the logical relativity of being. The whole idea that to be is to be the value of a variable makes no sense at all, at least concerning the ontologies of formal theories.

\section{REFERENCES}

BERRY, G. "Logic with Platonism" in Words and Objections, D. Davidson and J. Hintikka (eds.), p. 243-77, 1975

DAVIDSON, D and HINTINKA, J. (ed.) Words and Objections, Dordrecht: D. Reidel, 1975.

da COSTA, N. C. A. "Logic and ontology". Principia, 56, n.1, p. 279$298,2002$.

HAHN, L. and SCHILPP, P. The Philosophy of W.V. Quine. La Salle, ILL: Open Court, 1986.

JECH, T. Set Theory 2nd edition. New York: Springer-Verlag, 1997.

KUNEN, K. Set Theory. Dordrecht: Elsevier, 1980.

MADDY, P. Naturalism in Mathematics. Oxford: Clarendon Press, 1997. 
QUINE, W.V. "Reply to Parsons" In The Philosophy of W.V. Quine.L. Hahn and P. Schilpp (eds.), p. 396-403, 1986.

SMULLYAN, R. M. and FITTING, M. Set Theory and the Continuum Problem. Oxford: Clarendon Press, 1996. 